\title{
Age at menarche and risk of gestational diabetes mellitus: a population-based study in Xiamen, China
}

Liying Wang ${ }^{1,2+}$, Bing Yan ${ }^{1,2+}$, Xiulin Shi ${ }^{1,2}$, Haiqu Song ${ }^{1,2}$, Weijuan Su ${ }^{1,2}$, Bingkun Huang ${ }^{1,2}$, Yuxian Zhang ${ }^{1,2}$, Shunhua Wang ${ }^{1,2}$, Fuping LV $\mathrm{LV}^{1,2}$, Mingzhu Lin ${ }^{1,2}$ and Xuejun $\mathrm{Li}^{1,2^{*}}$

\begin{abstract}
Background: It has been reported that earlier age at menarche is associated with a higher risk for type 2 diabetes mellitus. However, the relationship between age at menarche and gestational diabetes mellitus is inconsistent across studies. We hypothesized that an earlier age at menarche would predict the gestational diabetes mellitus risk.

Methods: This was a population-based, retrospective cohort study of 70,041 women aged 18 to 53 years old, conducted between 2011 and 2018. The subjects were recruited from the Medical Birth Registry in Xiamen, China. Age at menarche was categorized as 8-12, 13, 14, 15, 16-19 years old. Logistic regression analysis and spline analysis was used to assess the risk of the menarche age group for gestational diabetes mellitus. Linear regression analysis was performed to evaluate independent associations between age at menarche and fasting plasma glucose and blood glucose 1 hour and 2 hours after a 75-g of glucose load between 24 and 28 weeks' gestation.

Results: The overall prevalence of GDM was $17.6 \%$. After adjustment for family history of diabetes, earlier age at menarche (8-12, and 13 years old) was associated with increased odds for GDM $(O R, 1.08 ; 95 \% \mathrm{Cl}, 1.02-1.15$, and $\mathrm{OR}, 1.07 ; 95 \% \mathrm{Cl}, 1.03-1.14$, respectively) compared with average age at menarche (14 years old). With further adjustment for pre-pregnancy body mass index, blood pressure, educational level, age at delivery, and hepatitis B surface antigen status, this association was attenuated (OR, 0.93, and $O R, 1.02$, respectively). Multivariable-adjusted spline regression models showed a linear dose-response association between age at menarche and GDM (P for nonlinearity, 0.203; P for linearity, 0.006). On linear regression analysis, earlier age at menarche was significantly associated with increased blood glucose one and 2 hours after a glucose load but not with the fasting plasma glucose.

Conclusions: As expected, early age at menarche was found to be associated with an increased risk of gestational diabetes mellitus. However, this association may be mediated by potential confounding factors other than age. An additional finding was that earlier menarche was significantly related with elevated pregnancy glucose concentrations after a glucose load.
\end{abstract}

Keywords: Body mass index, Gestational diabetes mellitus, Menarche, Pregnancy, Woman

\footnotetext{
* Correspondence: xmlixuejun@163.com

†Liying Wang and Bing Yan contributed equally to this work.

${ }^{1}$ Xiamen Diabetes Institute, Xiamen, China

2Department of Endocrinology and Diabetes, The First Affiliated Hospital,

Xiamen University, No.55 Zhenhai Road, Xaimen 361003, China
}

(c) The Author(s). 2019 Open Access This article is distributed under the terms of the Creative Commons Attribution 4.0 International License (http://creativecommons.org/licenses/by/4.0/), which permits unrestricted use, distribution, and reproduction in any medium, provided you give appropriate credit to the original author(s) and the source, provide a link to the Creative Commons license, and indicate if changes were made. The Creative Commons Public Domain Dedication waiver (http://creativecommons.org/publicdomain/zero/1.0/) applies to the data made available in this article, unless otherwise stated. 


\section{Background}

Gestational diabetes mellitus (GDM) is one of the most common pregnancy complications, affecting 9.3-19.7\% of all pregnancies in China [1-3]. With the increasing prevalence of GDM and given the strong link between GDM and type 2 diabetes, the identification of women who are at high risk of GDM has important public health and clinical significance. Menarche is an indicator of pubertal onset and the beginning of reproductive life in women. Studies have demonstrated an association between an earlier age at menarche and elevated fasting glucose, fasting insulin, insulin resistance, and the risk for type 2 diabetes [4-7], metabolic syndrome [8], all cause or cardiovascular disease mortality $[9,10]$, and breast cancer [11]. The precise mechanism by which early age at menarche may increase the risk of GDM is not clear. Some studies indicated that the early age at menarche was a risk factor of GDM [12-14]. Additionally, Early age at menarche has been associated with risk factors for GDM such as excessive pre-pregnancy obesity [15-17], insulin resistance [18], and hormonal changes $[19,20]$. However, the potential relationship of age at menarche and the risk of GDM have been inconclusive. Some studies founded a potential association $[14,21,22]$, whereas others show no association [23]. In this study, we examined whether there was an independent association between age at menarche and GDM risk, or with raised pregnancy glucose concentrations, in Chinese women.

\section{Methods}

\section{Study design and population}

This study was a retrospective evaluation of the association between age at menarche and GDM risk. The data were collected between January 2011 and March 2018 from the Medical Birth Registry of Xiamen (MBRX), China. The MBRX was established in 2007 that is based on compulsory notification of all live and stillbirths from 12 weeks' gestation. The MBRX contains information on maternal characteristics (age at menarche, maternal age, education, body mass index [BMI], obstetric history, etc.); pregnancy, labor, and delivery characteristics (gestational diabetes, gestational weight gain, gestational age at delivery, hypertension in pregnancy, etc.); and birth outcomes (fetal and neonatal death, birth weight, Apgar score at $5 \mathrm{~min}$, etc.).

The population in this cohort study was derived from the MBRX that was linked by individual record linkages to the Xiamen citizen health information system using the person-unique identification number assigned to each Xiamen citizen. All the study participants were over 18 years of age. Between 24 and 28 weeks' gestation, the mothers without diagnosed diabetes were advised to undergo a 75-g oral glucose tolerance test (OGTT) after fasting overnight. Participants were excluded who were known to have a multiple pregnancy or had received medical treatment for chronic diseases, such as oral glucocorticoids, thiazide diuretics, $\beta$-blockers, ACE inhibitors, or antiretroviral agents, before OGTT. From 2011 to 2018, there were 279,992 births recorded in this Register system. We restricted our cohort to 70,041 singletons born to pregnant women who underwent a 75-g OGTT and had menarche age records between March 1, 2011 and March 30, 2018. Study procedures were approved by the institutional review boards of the First Affiliated Hospital of Xiamen University, Xiamen, China.

\section{GDM assessment}

GDM cases were identified with the OGTT that conducted between 24 and 28 weeks' gestation, which is considered the optimal period to make this diagnosis. According to the 2014 National Health and Family Planning Commission of the People's Republic of China criteria, pregnant women were considered to have GDM if one of the following plasma glucose values was met or exceeded: $0 \mathrm{~h}, 5.1 \mathrm{mmol} / \mathrm{L} ; 1 \mathrm{~h}, 10.0 \mathrm{mmol} / \mathrm{L}$; or $2 \mathrm{~h}$, $8.5 \mathrm{mmol} / \mathrm{L}$, after a 75 -g glucose load. Even if the test was performed after 28 weeks, it was considered valid.

\section{Age at menarche}

This was defined as the age at the first menstrual period. The information on age at menarche was obtained by questionnaire: "At what age did you have your first menstrual period?". The MBRX summarized the data that came from the Women and Children Medical and Healthcare Centre of Xiamen. For our analysis, the age at menarche of participants was divided as: 8-12 years old, 13 years old, 14 years old, 15 years old, and 16-19 years old. The early age at menarche $(<13$ years) that consistent with the categories used in other research [24].

\section{Statistical analysis}

Analyses were restricted to the 70,041 women who recorded their menarche at a physiological age between 10 and 20 years. Continuous variables were expressed as means and standard deviations, whereas categorical variables were expressed as percentages. One way analysis of variance (ANOVA) was used to compare the means of the continuous variables, and the Chi-squared test was used to compare the proportions of the categorical variables. Logistic regression analysis was performed to estimate the odds ratios (OR) and 95\% confidence interval (CI) of GDM for each age at menarche group (8-12, 13, 14,15 , and 16-19 years old), with the median age at menarche (14 years) serving as the reference category, after adjusting for potential confounding and mediating factors. Linear regression analysis was conducted to 
evaluate independent associations between the age at menarche and fasting plasma glucose, one and 2 hours after a 75-g glucose load between 24 and 28 weeks' gestation. Significance tests were two-tailed and a $P$-value $<0.05$ was considered statistically significant. The data analysis for this article was generated using SPSS (version 19 of the SPSS system for the Windows $\times$ 64-based system) and SAS software (version 9.4, SAS Institute Inc. Cary, NC, USA.).

\section{Results}

Two hundred nine thousand nine hundred fifty-one participants were excluded, 70,041 participants were included. By comparing the main characteristics, we found no difference between the included and excluded population. The specific characteristics of the excluded and included groups are as following: age: $28.4 \pm 4.8$ vs. $28.4 \pm 4.1, P=0.291$; BMI: $21.1 \pm 3.0$ vs. $21.0 \pm 2.9, P=$ 0.236 ; plasma glucose, $4.5 \pm 0.5$ vs. $4.5 \pm 0.4, P=0.342$; Systolic blood pressure, $107.6 \pm 10.4$ vs. $107.5 \pm 10.7, P=$ 0.443 ; Diastolic blood pressure, $65.7 \pm 7.8$ vs. $65.6 \pm$ $7.9, P=0.551$; Education ( $\leq 9$ years), $24.1 \%$ vs. $23.5 \%$, $P=0.360$; Family history of diabetes, $2.7 \%$ vs. $2.6 \%$, $P=0.523$; Family history of hypertension, $6.4 \%$ vs. $6.4 \%, P=0.481$. The pregnant women included in our analysis had a mean (SD) age at menarche of 14.1 (1.6) years. The proportion of participants reporting menarche at ages at $8-12,13,14,15$, and $16-19$ years old was $10.4,24.6$, $30.0,19.5$, and $15.5 \%$, respectively. The characteristics and mean 75 -g oral glucose tolerance test plasma glucose levels of the participants at 24 to 28 weeks' gestation (based on age at menarche) are presented in Table 1.

Compared with earlier age at menarche (8-12 years old), women at a later age at menarche (16-19 years old) had a lower body mass index $\left(20.6 \pm 2.7 \mathrm{vs.} 21.7 \pm 3.0 \mathrm{~kg} / \mathrm{m}^{2}\right.$, $P<0.001)$, systolic blood pressure (107.3 \pm 10.6 vs. $108.3 \pm$
$10.6 \mathrm{mmHg}, P<0.001$ ), and diastolic blood pressure $(65.1 \pm 7.8$ vs. $66.4 \pm 7.9 \mathrm{mmHg}, P<0.001)$. A higher proportion of women with an earlier age at menarche had a family history of diabetes $(4.3 \%$ vs. $3.1 \%$ vs. $2.4 \%$ vs. $2.2 \%$ vs. $1.7 \%$, respectively, $P<0.001)$ and family history of hypertension $(7.5 \%$ vs. $6.0 \%$ vs. $5.2 \%$ vs. $5.1 \%$ vs. $4.5 \%$, respectively, $P<0.001$ ) than those with an average or late age at menarche, but a lower proportion had chronic hepatitis B infection $(6.4 \%$ vs. $8.5 \%$ vs. $8.2 \%$ vs. $9.1 \%$ vs. $9.2 \%$, respectively, $P<0.001)$ (Table 1).The results of the OGTT showed that women with an earlier age at menarche had higher fasting, one-, and two-hour plasma glucose levels than those with average or later age at menarche (all $P<0.001$ ) (Table 1).

In the present study, the overall prevalence of gestational diabetes mellitus was $17.6 \%$. The GDM prevalence, according to the age at menarche, was $10.4 \%$ (8-12 years old), $24.6 \%$ ( 13 years old), $30.0 \%$ (14 years old), $19.5 \%$ (15 years old), and $15.5 \%$ (16-19 years old). The crude GDM prevalence was highest in those with an earlier age at menarche. Table 2 presents the odds ratio (OR) for GDM by age at menarche. After adjustment for family history of diabetes, earlier age at menarche (8-12 years old) was associated with increased odds for GDM (OR 1.08 [95\% CI 1.02-1.15]) compared with average age at menarche ( 14 years old). The association was no longer significant (OR 0.93 [95\% CI $0.85-$ 1.03]) after additional adjustment for pre-pregnancy body mass index, blood pressure, educational level, age at delivery, and hepatitis B surface antigen (HBsAg) status. Furthermore, Age at menarche menopause was treated as a continuous variable, the results showed that the crude OR for GDM per 1 year older at menarche was 0.98 (95\% CI $0.96,0.99)$, however, these associations attenuated towards the null following adjustment for blood pressure and BMI (Table 3). In addition, multivariable-adjusted

Table 1 Clinical characteristics of subjects by age at menarche

\begin{tabular}{|c|c|c|c|c|c|c|}
\hline Age at menarche (year) & $\begin{array}{l}8-12 \text { years } \\
(n=7770,10.4 \%)\end{array}$ & $\begin{array}{l}13 \text { years } \\
(n=18,419,24.6 \%)\end{array}$ & $\begin{array}{l}14 \text { years } \\
(n=22,585,30.0 \%)\end{array}$ & $\begin{array}{l}15 \text { years } \\
(n=14,650,19.5 \%)\end{array}$ & $\begin{array}{l}16-19 \text { years } \\
(n=11,635,15.5 \%)\end{array}$ & $P$-value \\
\hline Age, year & $28.5 \pm 4.0$ & $28.3 \pm 4.0$ & $28.3 \pm 4.0$ & $28.4 \pm 4.2$ & $28.6 \pm 4.3$ & $<0.001^{*}$ \\
\hline Body mass index, BMI & $21.7 \pm 3.0$ & $21.2 \pm 3.0$ & $20.9 \pm 2.8$ & $20.9 \pm 2.8$ & $20.6 \pm 2.7$ & $<0.001^{*}$ \\
\hline Systolic blood pressure $(\mathrm{mmHg})$ & $108.3 \pm 10.6$ & $107.9 \pm 10.6$ & $107.4 \pm 10.6$ & $107.5 \pm 10.6$ & $107.3 \pm 10.6$ & $<0.001^{*}$ \\
\hline Diastolic blood pressure $(\mathrm{mmHg})$ & $66.4 \pm 7.9$ & $65.9 \pm 7.9$ & $65.5 \pm 7.9$ & $65.3 \pm 7.8$ & $65.1 \pm 7.8$ & $<0.001^{*}$ \\
\hline Education ( $\leq 9$ years) & $937(13.5 \%)$ & $3377(20.6) \%$ & $4271(23.7) \%$ & $3744(29.1 \%)$ & $3601(34.9 \%)$ & $<0.001^{*}$ \\
\hline Family history of diabetes & $333(4.3 \%)$ & $565(3.1 \%)$ & $544(2.4 \%)$ & $323(2.2 \%)$ & $201(1.7 \%)$ & $<0.001^{*}$ \\
\hline Family history of hypertension & $586(7.5 \%)$ & $1112(6.0 \%)$ & $1168(5.2 \%)$ & $757(5.1 \%)$ & $519(4.5 \%)$ & $<0.001^{*}$ \\
\hline HbsAg positive & $222(6.4 \%)$ & $785(8.5) \%$ & $874(8.2 \%)$ & $672(9.1 \%)$ & $1191(9.2 \%)$ & $<0.001^{*}$ \\
\hline Glucose 0 min OGTT (mmol/L) & $4.51 \pm 0.4$ & $4.50 \pm 0.4$ & $4.49 \pm 0.4$ & $4.48 \pm 0.39$ & $4.48 \pm 0.39$ & $<0.001^{*}$ \\
\hline Glucose $60 \mathrm{~min}$ OGTT (mmol/L) & $7.91 \pm 1.65$ & $7.85 \pm 1.69$ & $7.81 \pm 1.68$ & $7.80 \pm 1.67$ & $7.80 \pm 1.67$ & $<0.001^{*}$ \\
\hline Glucose 120 min OGTT (mmol/L) & $6.78 \pm 1.39$ & $6.71 \pm 1.38$ & $6.68 \pm 1.38$ & $6.67 \pm 1.37$ & $6.66 \pm 1.38$ & $<0.001^{*}$ \\
\hline
\end{tabular}


Table 2 Adjusted odds ratios (ORs) with associated 95\% confidence interval (Cl) of gestational diabetes mellitus

\begin{tabular}{|c|c|c|c|c|c|c|c|c|c|c|c|}
\hline & \multicolumn{10}{|c|}{ Age at menarche } & \multirow{3}{*}{$P_{\text {trend }}$} \\
\hline & \multicolumn{2}{|c|}{$\begin{array}{l}8-12 \text { years } \\
(n=7770)\end{array}$} & \multicolumn{2}{|c|}{$\begin{array}{l}13 \text { years } \\
(n=18,419)\end{array}$} & \multicolumn{2}{|c|}{$\begin{array}{l}14 \text { years } \\
(n=22,585)\end{array}$} & \multicolumn{2}{|c|}{$\begin{array}{l}15 \text { years } \\
(n=26,285)\end{array}$} & \multicolumn{2}{|c|}{$\begin{array}{l}16-19 \text { years } \\
(n=26,285)\end{array}$} & \\
\hline & OR & $95 \% \mathrm{Cl}$ & OR & $95 \% \mathrm{Cl}$ & $\mathrm{OR}$ & $95 \% \mathrm{Cl}$ & OR & $95 \% \mathrm{Cl}$ & $\mathrm{OR}$ & $95 \% \mathrm{Cl}$ & \\
\hline Crude model & 1.09 & $1.02-1.16$ & 1.08 & $1.02-1.13$ & 1 & referent & 0.97 & $0.92-1.02$ & 0.99 & $0.93-1.05$ & 0.001 \\
\hline Model 1 & 1.08 & $1.02-1.15$ & 1.07 & $1.03-1.14$ & 1 & referent & 0.97 & $0.92-1.02$ & 0.99 & $0.94-1.05$ & 0.001 \\
\hline Model 2 & 1.08 & $1.01-1.16$ & 1.10 & $1.05-1.17$ & 1 & referent & 0.97 & $0.92-1.02$ & 0.98 & $0.92-1.04$ & 0.001 \\
\hline Model 3 & 1.03 & $0.95-1.13$ & 1.07 & $0.99-1.14$ & 1 & referent & 0.96 & $0.89-1.03$ & 1.02 & $0.95-1.10$ & 0.113 \\
\hline Model 4 & 0.93 & $0.85-1.03$ & 1.02 & $0.95-1.08$ & 1 & referent & 0.97 & $0.91-1.05$ & 1.06 & $0.99-1.18$ & 0.094 \\
\hline
\end{tabular}

Model 1 adjusted for family history of diabetes

Model 2 adjusted for variables in model 1 plus education levels, age at delivery, chronic hepatitis B virus status

Model 3 adjusted for variables in model 2 plus systolic blood pressure, diastolic blood pressure

Model 4 adjusted for variables in model 3 plus pre-pregnancy BMI

spline regression models showed a linear dose-response association between age at menarche and GDM (P for nonlinearity, 0.203; P for linearity, 0.006) (Fig. 1). Meanwhile, linear regression analysis (Table 4) showed an inverse association between the age at menarche and fasting, one- and two-hour glucose levels. The relationship with fasting glucose was no longer significant after adjustment for family history of diabetes, education levels, age at delivery, chronic hepatitis B virus status, blood pressure, and pre-pregnancy BMI. Even after adjusting for possible confounding variables, age at menarche was significant and inversely associated with the one- and two-hour glucose levels.

\section{Discussion}

We observed two major findings in this study. On the one hand, earlier menarche tended to be associated with gestational diabetes mellitus after adjusting for age, but after further adjustment for pre-pregnancy body mass index, blood pressure, educational level, family history of diabetes, and hepatitis B surface antigen ( $\mathrm{HBsAg}$ ), this association was attenuated. Of which, many studies indicated a significant association between $\mathrm{HBsAg}$ positive and GDM [25-27]. Therefore, we adjusted the HBsAg factor that resulted in the association was weak in this

Table 3 Multivariable logistic of regression of gestational diabetes mellitus (Age at menarche as a continuous variable)

\begin{tabular}{llll}
\hline Age at menarche (year) & OR & $95 \% \mathrm{Cl}$ & $P$-value \\
\hline Crude model & 0.975 & $0.962-0.989$ & $<0.001$ \\
Model 1 & 0.979 & $0.966-0.993$ & $<0.001$ \\
Model 2 & 0.970 & $0.956-0.984$ & $<0.001$ \\
Model 3 & 0.987 & $0.970-1.005$ & 0.1627 \\
Model 4 & 1.002 & $1.000-1.041$ & 0.0610 \\
\hline
\end{tabular}

Model 1 adjusted for family history of diabetes

Model 2 adjusted for variables in model 1 plus education levels, age at delivery, chronic hepatitis B virus status

Model 3 adjusted for variables in model 2 plus systolic blood pressure, diastolic blood pressure

Model 4 adjusted for variables in model 3 plus pre-pregnancy BMI study. Meanwhile, later age at menarche may be not related with gestational diabetes mellitus. On the other hand, earlier age at menarche was significantly associated with increased one- and two-hour blood glucose after a glucose load at 24 to 28 weeks' gestation, even after adjusting for a number of confounding factors. The association may be mediated through age, blood pressure, and pre-pregnancy BMI between age at menarche and fasting plasma glucose at 24 to 28 weeks' gestation. To our knowledge, there are a few studies demonstrating a significant association between an earlier age at menarche and elevated glucose levels after a glucose load in China.

Meanwhile, we found that the prevalence of GDM $(17.6 \%)$ is higher than other populations in this study. One research reported that the total incidence of GDM in mainland of China was $14.8 \%$ [28]. A vast territory and a large population in China with significant differences in ethnicities, diets, lifestyles, and regions, and these factors may result in differences in prevalence of GDM reported in various regions. In addition, our study indicated that the average age at menarche $(14.1 \pm 1.6)$ is higher in this population than in many of the published studies. A study showed the overall mean age at menarche was 12.7 years in Korean girls [24]. Besides, another Chinese study expressed that mean age at menarche was $13.1 \pm 1.2$ years in Wuhan, a city located in Hubei province, China [22]. A large population in China with significant differences in ethnicities, diets, and lifestyles may lead to differences in mean age at menarche reported in various regions. As well, the BMI of categories in this research was lower than a U.S. study [29]. L.W. Chen et al., indicated that mean age at menarche at 13 years, the BMI of pre-pregnancy $\left(23.3 \pm 4.1 \mathrm{~kg} / \mathrm{m}^{2}\right)$ was higher than our study $\left(21.3 \pm 3.0 \mathrm{~kg} / \mathrm{m}^{2}\right)$. This significant difference was original from the difference of ethnics and smaller sample size compared with other study.

Whereas most studies have reported an increased risk of type 2 diabetes with earlier age at menarche, few 


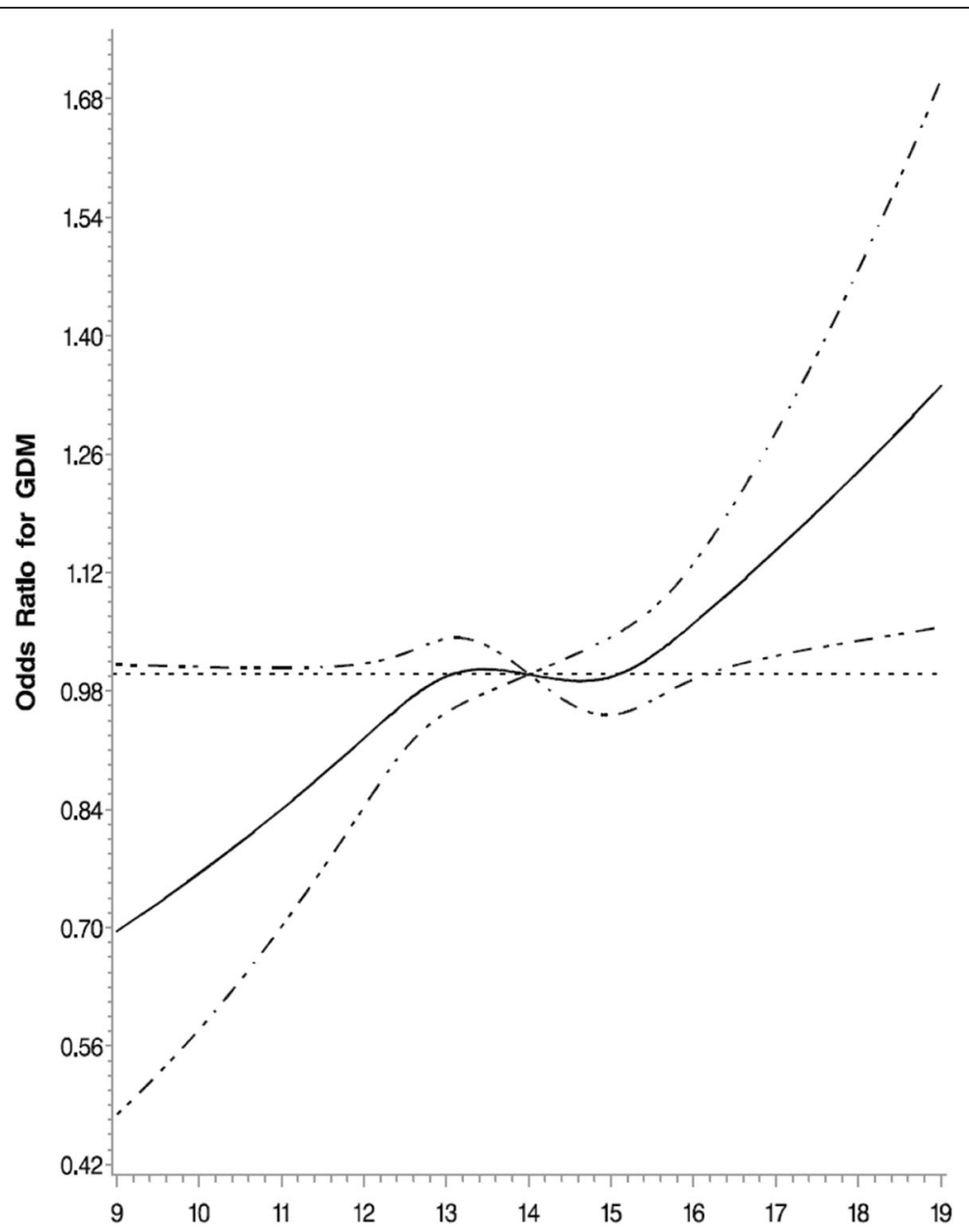

Fig. 1 Association of age at menarche and GDM. Odds ratios (ORs) and 95\% Cls derived from restricted cubic spline regression. Reference point is 14 years at menarche. ORs were adjusted for the same variables as model 4 in Table 2

studies have examined a possible association between age at menarche and the risk of GDM. These few studies have shown inconsistent results; most studies found that earlier age at menarche is associated with an increased risk of GDM [13, 14, 22, 29]. In addition, a meta-analysis showed that women with menarche at an early age ( $\leq$ 11 years) had a higher GDM risk with no significant heterogeneity between studies $\left(P=0.17 ; \quad \mathrm{I}^{2}=38\right)$ [13]. Whereas Dishi et al. found no such association [23]. Dishi et al. also suggested that an association of early menarche with the risk of GDM could differ that affected by pre-pregnancy BMI. In our study, this association was independent of age at delivery, but the association was significantly disappeared after further adjustment for the pre-pregnancy BMI. Both increased BMI and blood pressures are likely to be associated with insulin resistance in pregnancy which has also been shown to be negatively associated with age at menarche. However, our further research showed that the results of unadjusted variable of
BMI or BMI and blood pressure that indicated the earlier age at menarche was significantly associated with GDM.

Few studies have demonstrated that early age at menarche is linked to higher fasting, one-, and two-hour plasma glucose levels, even after adjustment for confounding factors [22, 30,31]. However, our results only showed a significant association existed between earlier age at menarche and stimulated glucose levels. The association was completely mediated by age at delivery, pre-pregnancy BMI, and blood pressure levels between age at menarche and fasting plasma glucose in pregnancy. Interestingly, our study indicated that the OGTT $60 \mathrm{~min}$ and $120 \mathrm{~min}$ glucose concentrations were higher at earlier age at menarche than mean or later age at menarche. It is noteworthy that multivariable linear regression showed that the coefficient was very small for association between glucose concentrations and age at menarche, despite the statistically significant $p$-values due to the large sample size. Studies reported that earlier 
Table 4 Multivariable linear regression of plasma glucose of OGTT

\begin{tabular}{cccc}
\hline Age at menarche (year) & Coefficient & SE & $P$-value \\
\hline Glucose 0 min OGTT (mmol/L) & & & \\
Model 1 & -0.004 & 0.001 & $<0.001$ \\
Model 2 & -0.009 & 0.002 & $<0.001$ \\
Model 3 & -0.006 & 0.002 & $<0.001$ \\
Model 4 & 0.004 & 0.002 & 0.059 \\
Glucose 60 min OGTT (mmol/L) & & \\
Model 1 & -0.030 & 0.002 & $<0.001$ \\
Model 2 & -0.030 & 0.004 & $<0.001$ \\
Model 3 & -0.020 & 0.008 & 0.021 \\
Model 4 & -0.016 & 0.004 & 0.040 \\
Glucose 120 min OGTT (mmol/L) & & \\
Model 1 & -0.030 & 0.005 & $<0.001$ \\
Model 2 & -0.032 & 0.005 & $<0.001$ \\
Model 3 & -0.023 & 0.007 & $<0.001$ \\
Model 4 & -0.021 & 0.005 & 0.003 \\
\hline
\end{tabular}

Model 1 adjusted for family history of diabetes

Model 2 adjusted for variables in model 1 plus education levels, age at delivery, chronic hepatitis B virus status

Model 3 adjusted for variables in model 2 plus systolic blood pressure, diastolic blood pressure

Model 4 adjusted for variables in model 3 plus pre-pregnancy BMI

age at menarche might be associated with adulthood higher estrogen levels and lower serum sex hormonebinging globulin levels among women [19], which could lead to higher OGTT $60 \mathrm{~min}$ and $120 \mathrm{~min}$ glucose levels. However, the KORA F4 study founded that young age at menarche was significantly related to higher fasting plasma glucose, and $120 \mathrm{~min}$ glucose concentrations [30]. Meanwhile, one published study showed a negative linear association between age at menarche and OGTT $60 \mathrm{~min}$ glucose concentrations in pregnancy [22]. Besides, a research elucidated that there was no significant association existed between menarche age and diabetes [32]. The sample sizes of populations and methods of statistical analysis may lead to aforementioned differences.

The strengths of our study included the large sample size and the GDM was diagnosed based on the oral glucose tolerance test. Nevertheless, several limitations also need to be acknowledged. Firstly, we were unable to obtain adequate information on childhood risk factors, which could influence both age at menarche and abnormal glucose metabolism. Secondly, because age at menarche was self-reported, misclassification may have occurred. Thirdly, this study was restricted to a medium-sized city in China with a relatively developed economy. However, data on income or job type was not collected. Therefore, the profile of socioeconomic status of the study population was not clearly known. And the results may not be generalizable to other socioeconomic groups. Fourthly, the results of this study were only applicable to Asian populations. Finally, it is inevitable that the linked and registered data are a potential source of bias in results of researches, yet researchers often discover it difficult to evaluate the extent of bias, because of the separation of linkage and analysis processes. Besides, we did not measure biomarkers for the mechanistic assessment of any possible relationships.

Taken together, this association between age at menarche and GDM should be further investigated in other study populations to identify our observations. Furthermore, the mechanistic researches are needed that elucidate underlying pathophysiologic mechanisms of observed correlation may help confirm therapeutic targets of a disorder related to significant offspring mortality. In addition, association between early age at menarche and GDM may help confirm women at high risk of GDM and adopt early prevention strategies.

\section{Conclusions}

In summary, we found an expected association between early age at menarche and increased GDM risk, but this association was less substantial when controlling for certain confounding factors. One significant additional finding from this study was that earlier menarche was inversely associated with pregnancy glucose concentrations following a glucose load. However, the coefficients for the outcomes were small. Future studies are needed to confirm our observations and/or expand on our results in other populations. Improved understanding of the relationship between the age at menarche and the risk of GDM will facilitate prevention and early detection.

\section{Abbreviations}

75-g OGTT: 75-g oral glucose tolerance test; BMI: Body mass index; FPG: Fasting plasma glucose; GDM: Gestational diabetes mellitus; IADPSG: the International Association of Diabetes and Pregnancy Study Groups; IDF: International Diabetes Federation; MBRX: Medical Birth Registry of Xiamen

\section{Acknowledgements}

We would like to thank all of the doctors, nurses, technicians, and patients involved at our centre for their dedication to the study.

\section{Funding}

The fund for this research was obtained from The First Affiliated Hospital of Xiamen University. It was used for collecting data and writing the manuscript.

Availability of data and materials

All data are held by Xiamen Diabetes Institute. It is available on request from Xiamen Diabetes Institute.

\section{Authors' contributions}

LW, BY, XS and XL performed the statistical analysis and wrote the manuscript; HS, WS, BH, YZ, SW, FL and ML participated in the data collection; XL participated in the design of the study and edited the manuscript. All authors read and approved the final manuscript. 


\section{Ethics approval and consent to participate}

This is a retrospective cohort study that did not require informed consent, which was approved by the ethics committee of the First Affiliated Hospital of Xiamen University that composed and worked in accordance with the Chinese GCP and relevant regulations. The application number was KYH2018-007. In addition, this study was carried out in accordance with the rules of the Declaration of Helsinki of 1975, revised in 2013.

\section{Consent for publication}

Not applicable.

\section{Competing interests}

The authors declare that they have no competing interests.

\section{Publisher's Note}

Springer Nature remains neutral with regard to jurisdictional claims in published maps and institutional affiliations.

Received: 21 November 2018 Accepted: 12 April 2019

Published online: 25 April 2019

\section{References}

1. Leng J, Shao P, Zhang C, Tian H, Zhang F, Zhang S, Dong L, Li L, Yu Z, Chan $\mathrm{JC}$, et al. Prevalence of gestational diabetes mellitus and its risk factors in Chinese pregnant women: a prospective population-based study in Tianjin, China. PLoS One. 2015:10(3):e0121029.

2. Zhu WW, Yang HX, Wang C, Su RN, Feng H, Kapur A. High prevalence of gestational diabetes mellitus in Beijing: effect of maternal birth weight and other risk factors. Chin Med J. 2017;130(9):1019-25.

3. Zhu WW, Yang HX, Wei YM, Yan J, Wang ZL, Li XL, Wu HR, Li N, Zhang MH, Liu XH, et al. Evaluation of the value of fasting plasma glucose in the first prenatal visit to diagnose gestational diabetes mellitus in China. Diabetes Care. 2013:36(3):586-90.

4. Wilson DA, Derraik JG, Rowe DL, Hofman PL, Cutfield WS. Earlier menarche is associated with lower insulin sensitivity and increased adiposity in young adult women. PLoS One. 2015;10(6):e0128427.

5. Stockl D, Doring A, Peters A, Thorand B, Heier M, Huth C, Stockl H Rathmann W, Kowall B, Meisinger C. Age at menarche is associated with prediabetes and diabetes in women (aged 32-81 years) from the general. population: the KORA F4 study. Diabetologia. 2012;55(3):681-8.

6. He C, Zhang C, Hunter DJ, Hankinson SE, Buck Louis GM, Hediger ML, Hu FB. Age at menarche and risk of type 2 diabetes: results from 2 large prospective cohort studies. Am J Epidemiol. 2010;171(3):334-44.

7. Yang L, Li L, Peters SAE, Clarke R, Guo Y, Chen Y, Bian Z, Sherliker P, Yin J, Tang $Z$, et al. Age at menarche and incidence of diabetes: a prospective study of 300,000 women in China. Am J Epidemiol. 2018:187(2):190-8.

8. Glueck CJMJ, Wang P, Woo JG. Early and late menarche are associated with oligomenorrhea and predict metabolic syndrome 26 years later. Metab Clin Exp. 2013;62:1597-606

9. Charalampopoulos DMA, Elks CE, Ong KK. Age at menarche and risks of allcause and cardiovascular death: a systematic review and meta-analysis. Am J Epidemiol. 2014;180:29-40.

10. Canoy DBV, Balkwill A, Wright FL, Kroll ME, Reeves GK, Green J, Cairns BJ, Million Women Study Collaborators. Age at menarche and risks of coronary heart and other vascular diseases in a large UK cohort. Circulation. 2015;131: 237-44.

11. Cancer. CGoHFiB. Menarche, menopause, and breast cancer risk: individual participant meta-analysis, including 118964 women with breast cancer from 117 epidemiological studies. Lancet Oncol. 2012:13:1141-51.

12. Xiao Y, Chen R, Chen M, Luo A, Chen D, Liang Q, Cai Y, Chen L, Zhao X. Age at menarche and risks of gestational diabetes mellitus: a meta-analysis of prospective studies. Oncotarget. 2018;9(24):17133-40.

13. Sun X, Yang L, Pan J, Yang H, Wu Y, Chen Z, Chen X, Mu L. Age at menarche and the risk of gestational diabetes mellitus: a systematic review and meta-analysis. Endocrine. 2018;61(2):204-9.

14. Schoenaker D, Mishra GD. Association between age at menarche and gestational diabetes mellitus: the Australian longitudinal study on Women's health. Am J Epidemiol. 2017:185(7):554-61.

15. Laitinen J, Power C, Jarvelin MR. Family social class, maternal body mass index, childhood body mass index, and age at menarche as predictors of adult obesity. Am J Clin Nutr. 2001;74(3):287-94.
16. Pierce $\mathrm{MB}$, Leon DA. Age at menarche and adult BMI in the Aberdeen children of the 1950s cohort study. Am J Clin Nutr. 2005;82(4):733-9.

17. Freedman DKL, Serdula M, Dietz W, Srinivasan S, Berenson G. The relation of menarcheal age to obesity in childhood and adulthood: the Bogalusa heart study. BMC Pediatr. 2003;3:3 Epub 2003 Apr 30.

18. Feng Y, Hong X, Wilker E, Li Z, Zhang W, Jin D, Liu X, Zang T, Xu X. Effects of age at menarche, reproductive years, and menopause on metabolic risk factors for cardiovascular diseases. Atherosclerosis. 2008;196(2):590-7.

19. Apter D, Reinila M, Vihko R. Some endocrine characteristics of early menarche, a risk factor for breast cancer, are preserved into adulthood. Int J Cancer. 1989:44(5):783-7.

20. Bartha JL, Comino-Delgado R, Romero-Carmona R, Gomez-Jaen MC. Sex hormone-binding globulin in gestational diabetes. Acta Obstet Gynecol Scand. 2000;79(10):839-45

21. Shen Y, Hu H, Taylor BD, Kan H, Xu X. Early menarche and gestational diabetes mellitus at first live birth. Matern Child Health J. 2017;21(3):593-8.

22. Li H, Shen L, Song L, Liu B, Zheng X, Xu S, Wang Y. Early age at menarche and gestational diabetes mellitus risk: results from the healthy baby cohort study. Diabetes Metab. 2017:43(3):248-52.

23. Dishi M, Enquobahrie DA, Abetew DF, Qiu C, Rudra CB, Williams MA. Age at menarche, menstrual cycle characteristics and risk of gestational diabetes. Diabetes Res Clin Pract. 2011:93(3):437-42.

24. Lee MH, Kim SH, Oh M, Lee KW, Park MJ. Age at menarche in Korean adolescents: trends and influencing factors. Reprod Health. 2016;13(1):121.

25. Tan J, Liu X, Mao X, Yu J, Chen M, Li Y, Sun X. HBsAg positivity during pregnancy and adverse maternal outcomes: a retrospective cohort analysis. J Viral Hepat. 2016;23(10):812-9.

26. Tse KY, Ho LF, Lao T. The impact of maternal HBsAg carrier status on pregnancy outcomes: a case-control study. J Hepatol. 2005;43(5):771-5.

27. Lao TT, Tse KY, Chan LY, Tam KF, Ho LF. HBsAg carrier status and the association between gestational diabetes with increased serum ferritin concentration in Chinese women. Diabetes Care. 2003;26(11):3011-6.

28. Gao C, Sun X, Lu L, Liu F, Yuan J. Prevalence of gestational diabetes mellitus in mainland China: a systematic review and meta-analysis. J Diabetes Investig. 2019:10(1):154-62.

29. Chen L, Li S, He C, Zhu Y, Buck Louis GM, Yeung E, Hu FB, Zhang C. Age at menarche and risk of gestational diabetes mellitus: a prospective cohort study among 27,482 women. Diabetes Care. 2016;39(3):469-71.

30. Stockl D, Meisinger C, Peters A, Thorand B, Huth C, Heier M, Rathmann W, Kowall B, Stockl H, Doring A. Age at menarche and its association with the metabolic syndrome and its components: results from the KORA F4 study. PLoS One. 2011;6(10):e26076.

31. Chen L, Zhang C, Yeung E, Ye A, Mumford SL, Wactawski-Wende J, Schisterman EF. Age at menarche and metabolic markers for type 2 diabetes in premenopausal women: the BioCycle study. J Clin Endocrinol Metab. 2011;96(6):E1007-12.

32. Qiu C, Chen H, Wen J, Zhu P, Lin F, Huang B, Wu P, Lin Q, Lin Y, Rao H, et al. Associations between age at menarche and menopause with cardiovascular disease, diabetes, and osteoporosis in Chinese women. J Clin Endocrinol Metab. 2013;98(4):1612-21.

Ready to submit your research? Choose BMC and benefit from:

- fast, convenient online submission

- thorough peer review by experienced researchers in your field

- rapid publication on acceptance

- support for research data, including large and complex data types

- gold Open Access which fosters wider collaboration and increased citations

- maximum visibility for your research: over $100 \mathrm{M}$ website views per year

At $\mathrm{BMC}$, research is always in progress.

Learn more biomedcentral.com/submissions 